\title{
GLOBAL BUNDLE ADJUSTMENT WITH VARIABLE ORIENTATION POINT DISTANCE FOR PRECISE MARS EXPRESS ORBIT RECONSTRUCTION
}

\author{
J. Bostelmann*, C. Heipke \\ Institute of Photogrammetry and GeoInformation (IPI) \\ Leibniz Universität Hannover \\ Nienburger Str.1, D-30167 Hannover, Germany \\ bostelmann@ipi.uni-hannover.de
}

Commission IV, WG IV/8

KEY WORDS: Planetary, Mapping, Mars, HRSC, Bundle Adjustment, Orbit Modeling

\begin{abstract}
:
The photogrammetric bundle adjustment of line scanner image data requires a precise description of the time-dependent image orientation. For this task exterior orientation parameters of discrete points are used to model position and viewing direction of a camera trajectory via polynomials. This paper investigates the influence of the distance between these orientation points on the quality of trajectory modeling. A new method adapts the distance along the trajectory to the available image information. Compared to a constant distance as used previously, a better reconstruction of the exterior orientation is possible, especially when image quality changes within a strip.

In our research we use image strips of the High Resolution Stereo Camera (HRSC), taken to map the Martian surface. Several experiments on the global image data set have been carried out to investigate how the bundle adjustment improves the image orientation, if the new method is employed. For evaluation the forward intersection errors of 3D points derived from HRSC images, as well as their remaining height differences to the MOLA DTM are used.

In $13.5 \%$ ( 515 of 3,828$)$ of the image strips, taken during this ongoing mission over the last 12 years, high frequency image distortions were found. Bundle adjustment with a constant orientation point distance was able to reconstruct the orbit in 239 (46.4\%) cases. A variable orientation point distance increased this number to $507(98.6 \%)$.
\end{abstract}

\section{INTRODUCTION}

For the photogrammetric processing of line scanner imagery some characteristic features have to be considered in the geometric modeling. Different from frame cameras images are not taken at a single moment. The image strips of line scanners are created line by line and are thus time-dependent. Each line or, in case of multiline cameras, set of lines has its own exterior orientation (EO) describing the position and attitude of the camera. Because of the continuous imaging process, the EO of neighboring lines is highly correlated. It is therefore possible to reduce the number of parameters in the model and use interpolated EO values for the majority of image lines. To accomplish this task by polynomial interpolation various formulations of the model are possible: Beside a simple linear interpolation (Hofmann et al., 1982), single low or higher order polynomials are used (Poli, 2005) as well as piecewise smooth polynomials like e.g. splines (Kornus, 1999), (Weser et al., 2008). For satellite imagery the physical model of a Kepler orbit can be used (Ohlhof, 1996). Very common is also the use of rational polynomial coefficients (RPCs) (Dowman and Dolloff, 2000), (Hu and Tao, 2002), (Fraser and Hanley, 2003).

In this work we discuss the modeling with cubic piecewise Lagrange polynomials for the EO of HRSC images. The High Resolution Stereo Camera is the photogrammetric imaging instrument on ESA's Mars Express mission (Neukum et al., 2004). This multi-line sensor with five panchromatic and four multi-spectral CCD lines was developed by the German Aerospace Center (DLR). It is the first camera in planetary research especially designed for photogrammetric mapping purposes (Albertz et al., 2005). The HRSC images the Martian surface with a resolution of up to $10 \mathrm{~m}$ per pixel. The along-track stereo capability of the camera delivers image strips with three-dimensional information, which covers nearly the entire planet (Gwinner et al., 2016).

${ }^{*}$ Corresponding author
Every single strip allows the generation of high resolution digital terrain models (DTMs) (Gwinner et al., 2009), (Heipke et al., 2007) and orthoimages (Gwinner et al., 2010). Radiometrically calibrated images as well as DTMs and orthoimages are regularly released to the NASA Planetary Data System (PDS) and the ESA Planetary Science Archive (PSA) (Jaumann et al., 2007) and are used by a large number of scientist e.g. for geological analysis. A summary of the scientific results of the experiment has been published in (Jaumann et al., 2015).

When imaging with the HRSC is performed the camera typically records image strips for all nine channels with a width of 5,184 pixels simultaneously. The length of the strips depends on the recording duration and varies broadly. On average the strips have 50,000 image lines per channel. About 5\% are shorter than 20,000 and about 5\% are longer than 100,000 image lines. Because of data storage and transfer limitations, as common for planetary missions, normally only the nadir channel retains its full resolution. The four other panchromatic channels are systematically reduced by a factor of 4 or 16 , the color channels even more. Additionally, image compression is applied.

After transfer of the HRSC images to the ground station, a radiometric calibration is applied (Scholten et al., 2005). The following reconstruction of the image orientation parameters is divided into two parts. After tie point generation via image matching (Schmidt, 2008) a two-stage bundle adjustment is carried out (Spiegel, 2007a), (Bostelmann et al., 2012). Within the bundle adjustment first, and this is the topic of this paper, the EO of each strip is reconstructed individually. This EO is then used in a block adjustment (Bostelmann and Heipke, 2014) to reduce inconsistencies between the strips. Afterwards DTMs are created and used for an ortho-rectification, either as single strips or as regional mosaics. For latter a radiometric adaption is applied within the mosaicing process (Michael et al., 2016). 
In the following the mathematical model of the bundle adjustment with its adaptations to the HRSC imagery is described. Section 3 depicts the setup and results of multiple experiments to demonstrate the benefits of bundle adjustment. Conclusions and an outlook on future work are given in the last section.

\section{MATHEMATIC MODEL OF THE BUNDLE ADJUSTMENT}

In this section we give a short summary of the mathematical model used for bundle adjustment. This model, uses orientation points (OP) and has been developed and refined over the years (Hofmann et al., 1982), (Müller, 1991), (Strunz, 1993), (Ohlhof, 1996), (Kornus, 1999), (Spiegel, 2007b) (Bostelmann and Heipke, 2011). A DTM derived from NASA's Mars Orbiter Laser Altimeter (MOLA) data (Smith et al., 2001) is used as reference.

\subsection{Functional Model}

A number of unknown parameters of this model are to be estimated in the adjustment. We put these unknowns into a vector $\mathbf{U}$ and arrange them into three groups, where each of these groups has a different functional model:

$$
\mathbf{U}=\left(\mathbf{U}_{P}, \mathbf{U}_{O}, \mathbf{U}_{B D}\right)^{T}
$$

These groups are:

1. The three-dimensional coordinates $X_{i}, Y_{i}, Z_{i}$ describe the position in object space of each tie point $P_{i}$ :

$$
\mathbf{U}_{P_{i}}=\left(X_{P_{i}}, Y_{P_{i}}, Z_{P_{i}}\right)
$$

2. Six parameters for the EO for each orientation point $O_{m}$ describe the position of the perspective center $X_{O_{m}}, Y_{O_{m}}$, $Z_{O_{m}}$ and the rotation between the camera and the object coordinate system given by the elements of rotation $\varphi_{O_{m}}$, $\omega_{O_{m}}, \kappa_{O_{m}}$ :

$$
\mathbf{U}_{O_{m}}=\left(X_{O_{m}}, Y_{O_{m}}, Z_{O_{m}}, \varphi_{O_{m}}, \omega_{O_{m}}, \kappa_{O_{m}}\right)
$$

3. Six parameters for a bias $X_{B}, Y_{B}, Z_{B}, \varphi_{B}, \omega_{B}, \kappa_{B}$ and six parameters for a drift $X_{D}, Y_{D}, Z_{D}, \varphi_{D}, \omega_{D}, \kappa_{D}$ allow to describe a systematic displacement of the strip:

$$
\mathbf{U}_{B D}=\left(X_{B}, \ldots, \kappa_{B}, X_{D}, \ldots, \kappa_{D}\right)
$$

The total number of unknowns $n_{U}$ for the bundle adjustment is accordingly dependent on the number of tie points $n_{P}$ and OP $n_{O}$ :

$$
n_{U}=3 \cdot n_{P}+6 \cdot n_{O}+12
$$

The observation equation of the least squares adjustment can be written as:

$$
\mathbf{v}=f(\mathbf{U})-\mathbf{z}
$$

were $\mathbf{z}$ describes the observations and $\mathbf{v}$ the residuals.

For a combined adjustment of HRSC image strips and the MOLA DTM, used as ground control information, four types of observation equations are used:

1. The coordinates of a tie point $P_{i}$ are measured in an image strip $s$. The transformation between these pixel coordinates and the image coordinates $\mathbf{z}_{c, i, s}=\left(x_{i}, y_{s}\right)$ acquired at time $t_{j}$ is described by the interior orientation $\mathrm{IO}_{s}$. The observation equations for the image coordinates are given by:

$$
\mathbf{v}_{c, i, s}=f\left(\mathbf{U}_{P_{i}}, \mathbf{U}_{O_{j}}\right)-\mathbf{z}_{c, i, s}
$$

$\mathbf{U}_{O_{j}}=\left(X_{O_{j}}, Y_{O_{j}}, Z_{O_{j}}, \varphi_{O_{j}}, \omega_{O_{j}}, \kappa_{O_{j}}\right)$ describes the EO at $t_{j}$. These parameters are interpolated from the $\mathrm{EO}$ of the neighboring OP.

For cubic Lagrange polynomials as used in our work four OP are necessary: $O_{m-1}, O_{m}, O_{m+1}$ and $O_{m+2}$. This allows to directly use the $\mathrm{EO}$ of the $\mathrm{OP}$ as sampling points:

$$
\mathbf{U}_{O_{j}}=\sum_{k=m-1}^{m+2} \mathbf{U}_{O_{k}} \prod_{l=m-1, l \neq k}^{m+2} \frac{t_{j}-t_{l}}{t_{k}-t_{l}}
$$

where $t_{m-1}<t_{m} \leq t_{j}<t_{m+1}<t_{m+2}$ are the image lines corresponding to the two previous and two next OP. How the position of the $\mathrm{OP}$ is defined will be explained later.

The observation function $f$ in (7) represents the extended collinearity equations. The interior orientation is assumed to be known and constant.

2. For the six EO parameters at each OP $O_{m}$ (3) the observations $\mathbf{z}_{O_{m}}$ are taken from the nominal EO provided by systematic pre-processing. Systematic errors are considered by $\mathbf{U}_{B D}=\left(\mathbf{U}_{B}, \mathbf{U}_{D}\right)^{T}$ :

$$
\mathbf{v}_{O_{m}}=\mathbf{U}_{O_{m}}+\mathbf{U}_{B}+t_{m} \cdot \mathbf{U}_{D}-\mathbf{z}_{O_{m}}
$$

3. In the absence of real observations for the bias and drift parameters pseudo observations $\mathbf{z}_{B D}=0$ are used:

$$
\mathbf{v}_{B D}=\mathbf{U}_{B D}-\mathbf{z}_{B D}=\mathbf{U}_{B D}
$$

4. The MOLA DTM is introduced as control information by adding a height difference as a pseudo observation $z_{H_{i}}=$ 0 . This formulates the assumption that in object space each HRSC tie point $P_{i}=\left(X_{P_{i}}, Y_{P_{i}}, Z_{P_{i}}\right)$ lies on the MOLA surface $Z_{M}\left(X_{P_{i}}, Y_{P_{i}}\right)$.

For the functional description a bi-linear interpolation is used, where $\left(X_{M_{q}}, Y_{M_{q}}, Z_{M_{q}}\right)$ are the coordinates of four neighboring MOLA DTM grid points $M_{1}, M_{2}, M_{3}, M_{4}$, given a DTM grid width of $d$ :

$$
\begin{aligned}
v_{H_{i}} & =Z_{P_{i}}-Z_{M}\left(X_{P_{i}}, Y_{P_{i}}\right)-z_{H_{i}} \\
& =Z_{P_{i}}-Z_{M}\left(X_{P_{i}}, Y_{P_{i}}\right)
\end{aligned}
$$

with:

$$
\begin{gathered}
Z_{M}\left(X_{P_{i}}, Y_{P_{i}}\right)= \\
\sum_{q=1}^{4}\left(1-\frac{X_{P_{i}}-X_{M_{q}}}{d}\right) \cdot\left(1-\frac{Y_{P_{i}}-Y_{M_{q}}}{d}\right) \cdot Z_{M_{q}}
\end{gathered}
$$

Given this functional model a robust bundle adjustment is carried out iteratively to compute optimized parameters for the EO of the imagery. 


\subsection{Stochastic model}

Essential for the bundle adjustment are the weights of the observations in the stochastic model for each particular type of observation:

- The image coordinates of the tie points are always introduced with an apriori standard deviation of $\sigma_{c}=1 \mu m=$ $1 / 7$ pixel.

- The observations of rotation, described by three angles at each OP are introduced with an a priori standard deviation of $\sigma_{\varphi \omega \kappa}=0.028 \mathrm{gon}$.

- Pseudo observations for bias allowing a translation of the whole strip are introduced with $\sigma_{B, X Y Z}=1000 \mathrm{~m}$.

- A drift for the height component of the EO is also permitted by setting $\sigma_{D, Z}=0.01 \mathrm{~m} /$ image line.

- For $\mathrm{X}$ and $\mathrm{Y}$ no drift is allowed: $\sigma_{d r i f t, X Y}=0$.

- For the orientation angles neither bias nor drift are considered: $\sigma_{B D, \varphi \omega \kappa}=0$

- The pseudo observation of the height difference for the DTM constraint is weighted with $\sigma_{M}=100 \mathrm{~m}$.

These values have been chosen according available information about the accuracy of the instruments and on the basis of empirical tests (Spiegel, 2007b).

\subsection{Orientation point distance}

To reliably estimate the six unknown parameters of the EO at each OP a sufficient number of tie points in the area between two $\mathrm{OP}$ is needed. In our previous work the distance (OPD, measured in sec) between all OP was defined by a single value. This constant orientation point distance (cOPD) required to increase the distance to a value, that all OP could reliably estimated. By introducing a variable distance between the OP (vOPD) it is possible to adapt the distance individually to the tie point density.

Our current implementation operates as follows: The first OP corresponds to the first image line. A minimum distance between the $O P \min _{O P D}$ defines the second OP. If the number of tie points between these two OP is lower than a value $\min _{T P}$ the OPD is increased by adding $\min _{O P D}$ until $\min _{T P}$ is reached. The distance between all following OP is determined in the same way. As a consequence the number of OP $\mathrm{n}_{O}$ depends also on the number and distribution of the tie point observations:

$$
n_{O}=f\left(\mathbf{z}_{c}, \min _{O P}, \min _{O P D}\right)
$$

For a systematic processing of large data sets the whole process, including pre-processing, tie point matching, all runs and iterations of the bundle adjustment and the evaluation is fully automated. It requires about $2 \mathrm{~h}$ computing time for each strip on average.

\section{EXPERIMENTS AND RESULTS}

To validate the proposed method of modeling the EO in the bundle adjustment, a set of experiments has been conducted. In the first part of this section the influence of the number of tie points is exemplarily analyzed on a single image strip. Then, the cOPD is compared to the vOPD and finally the bundle adjustment results of the global HRSC data set are presented.

\subsection{Tie point density}

In general, when modeling an orbit as described in section 2, the level of detail of the model depends on the number of OP. In some of the orbits the camera was affected by oscillations of the space craft during the imaging process. These oscillations affect the accuracy of the photogrammetric object point determination severely, as shown below (Fig. 2). They are believed to come from a periodic movement of the solar panels and have a constant frequency of about $0.12 \mathrm{~Hz}$ (Gwinner et al., 2015). Therefore, a corresponding $\max _{O P D}=4 s$ is needed.

For the bundle adjustment a suitable proportion between the number of OP and tie points is needed. Considering blunders, a minimum number of $\min _{T P}=50$ tie points between two OP have empirically proven to be suitable. The minimum distance between two OP was restricted to $\min _{O P D}=1 \mathrm{sec}$. This limit was set to avoid an unnecessarily high number of OP. These values were used for all of the results presented in the following.

To fulfill these two requirements $\left(\min _{T P}=50\right.$, and $\max _{O P D}=$ $4 s$ ), a sufficient tie point density must be available in the images. This density depends on the success rate of image matching. Fig. 1 shows a typical example where a low contrast area results in a low tie point density. From left to right the number of tie points increases but the center area remains nearly uncovered.

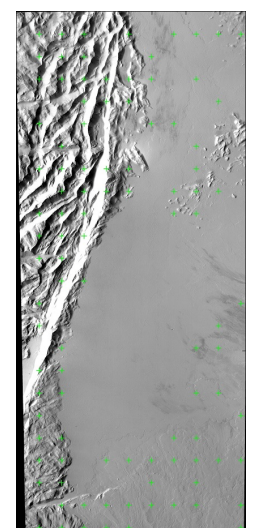

CP: 1,000

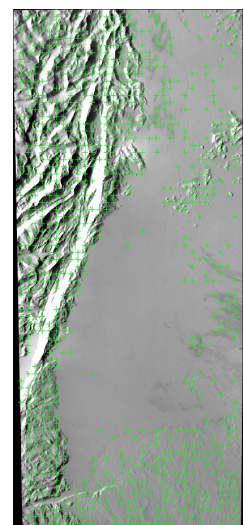

CP: 10,000

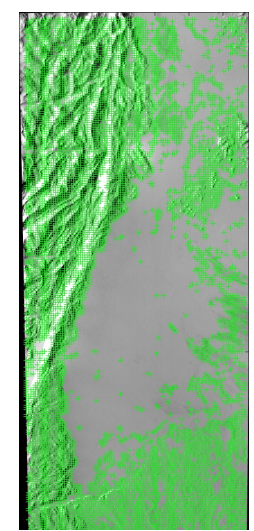

CP: 100,000
Figure 1: Increasing number of candidate tie points (CP). Green: successfully matched tie points. Background: part of the HRSC nadir image taken in orbit number 3,148

We performed a series of bundle adjustment runs with different numbers of tie points to better understand the general relationships between tie point density, OPD and a resulting mean object point accuracy from an experimental point of view.

All tests were carried out on a reasonably representative data set of 16 image strips. The goal was to find the required value for the tie point density, which should be used to process the global HRSC data set and allow an accurate orbit reconstruction with different image quality. One of these 16 strips is taken here as an example. The images were taken in orbit number 3,148 in 2006. The size of the Nadir image is 5,168x72,264 pixel. This orbit shows the typical behavior for a strip, where the bundle adjustment previously failed. It contains areas with locally very few tie points and $\mathrm{EO}$ with oscillations at the same time.

Table 1 compares six processing runs with different number of candidate tie points $(\mathrm{CP})$. The success rate of the image matching was about $70 \%$. The number of OP increases with the number of tie points (TP), and because of a better orbit modeling the mean intersection error (MIE) decreases. 


$\begin{array}{rrrrrr}\text { CP } & \text { TP } & \text { OP } & \text { mOPD[sec] } & \text { MIE[m] } & \text { Fig. 2 } \\ 100000 & 72540 & * 49 & 5.72 & 19.48 & \text { c) } \\ 1000 & 713 & 44 & 6.38 & 15.2 & \text { d) } \\ 2000 & 1459 & 80 & 3.51 & 10.77 & \text { e) } \\ 8000 & 6383 & 205 & 1.37 & 7.74 & \text { f) } \\ 12000 & 8756 & 220 & 1.28 & 6.52 & \text { g) } \\ 100000 & 72540 & 262 & 1.07 & 6.29 & \text { h) }\end{array}$

Table 1: Bundle adjustment of HRSC images: different number of tie points (TP) result in different mean orientation point distance (mOPD), * with cOPD, else vOPD

In Fig. 2 the forward intersection error for different numbers of tie points is shown with color-coded error maps. The plots were created by using the same, independently obtained set of about 100,000 tie points. a) shows an ortho-image of the nadir channel. b) was created by using the nominal EO. For c) 100,000 candidate tie points were used for a bundle adjustment with a cOPD. For d), e), f), g), and h) the adjusted EO of the test runs with a vOPD were used.

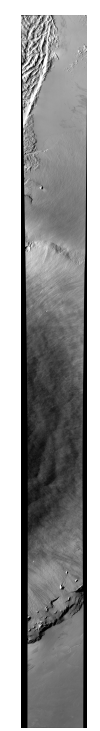

a)

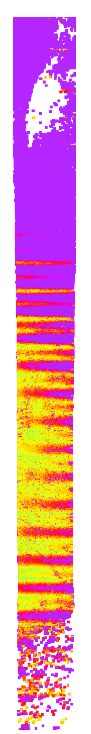

b)

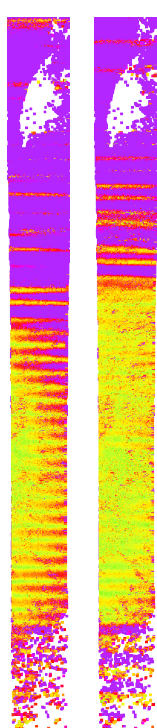

c)

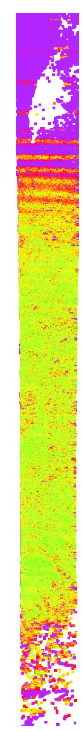

d) e)

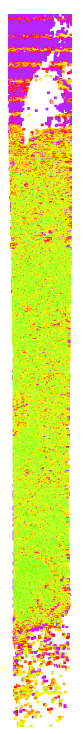

f)

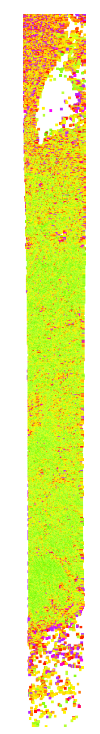

g)

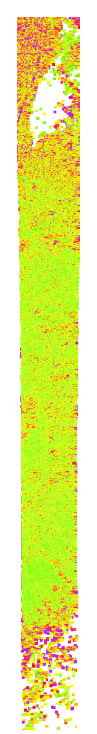

h)

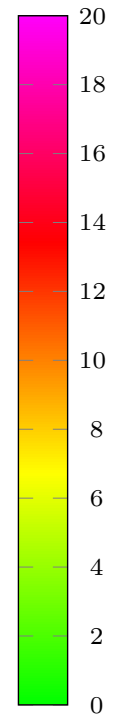

$[\mathrm{m}]$
Figure 2: Forward intersection error [m] of HRSC object points with oscillation effects reduced via bundle adjustment (compare Tab. 1)

It is visible that in Fig. $2 \mathrm{~g}$ ) with 12,000 tie points, the oscillations are quite well modeled and the intersection error is consequently low. Using more tie points, e.g. 100,000 as in Fig. 2 h) improves the EO only marginally. For a more detailed comparison the resulting numbers of constant and variable OPD are depicted in Fig. 3, 4 and 5 using a larger number of test runs. With a vOPD the number of OP becomes more or less constant at about 15.000 tie points (Fig. 3). The reason for that is, that the minimum requested distance $\min _{O P D}$ between most of the OP is reached. With a cOPD the number of OP only increases slowly, because of the areas with low tie point density, where $\min _{T P}=50$ disables a shorter OPD. Not surprisingly the mean OPD (Fig. 4) is negatively correlated to the number of OP. Note that for a cOPD the mean naturally equals the actual distances between all OP.

Fig. 5 depicts how the number of tie points affects the mean intersection error. Using more than 10.000 tie points just slightly improve the average object point precision. It is of course reasonable to link the number of tie points to the length of the image

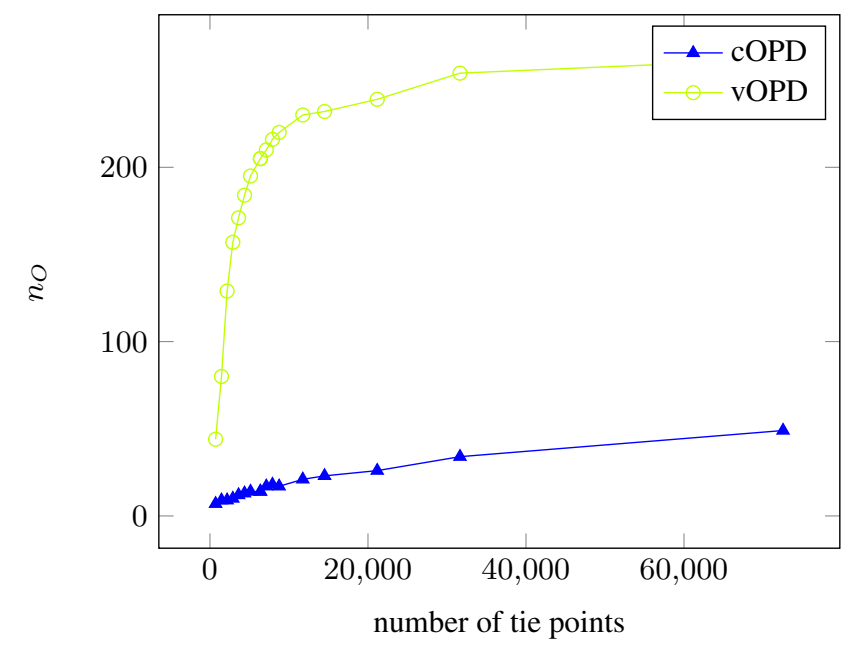

Figure 3: Number of orientation points $n_{O}$ increases with the number of tie points: Comparison between a constant and variable orientation point distance

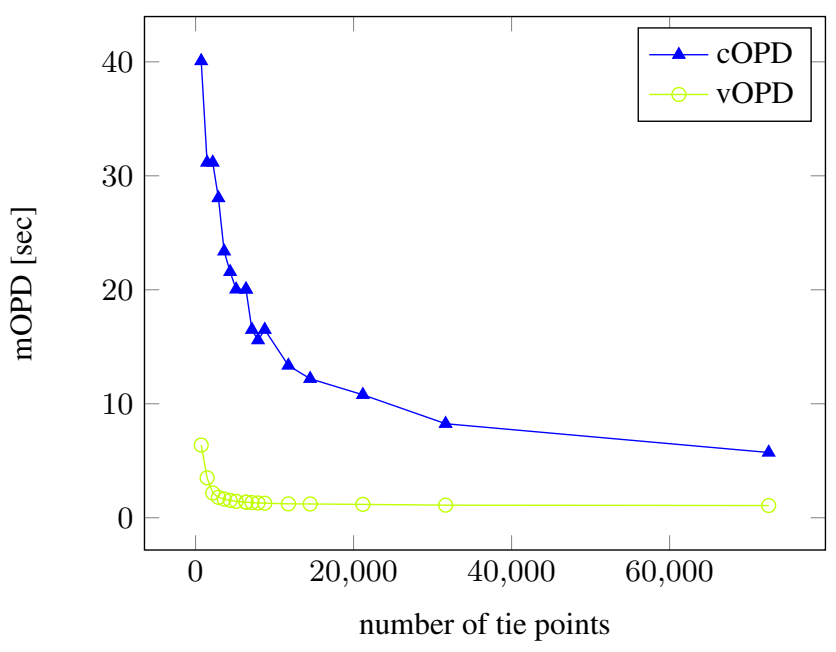

Figure 4: Mean orientation point distance (mOPD) decreases with the number of tie points: Comparison between a constant and variable orientation point distance

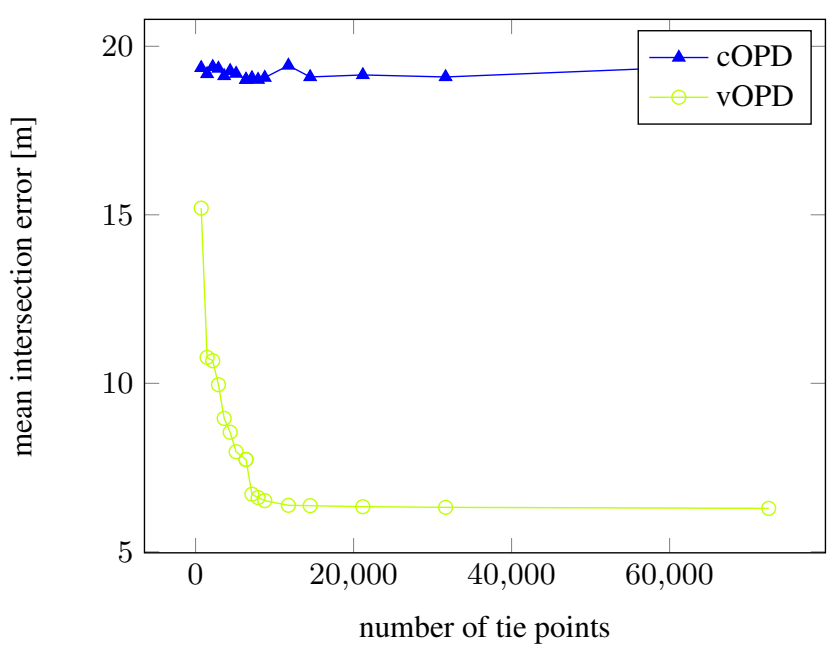

Figure 5: Mean intersection error decreases with the number of tie points: Comparison between a constant and variable orientation point distance 
strips. Using the 16 test strips we observed empirically, that a quarter of the number of image lines is generally sufficient for the number of tie points. In case of the results presented above from orbit 3,148 the number of image lines was 72,264. Although this strip contains oscillations and texture-less areas, one quarter $(18,066)$, is clearly a sufficient number of tie points (see Fig. 5). For that reason we used the number of image lines to set the number of tie points for a systematic processing of all HRSC image strips. The results are presented below.

\subsection{Oscillations in HRSC data}

By a systematic visual inspection of the evaluation results from all 3,828 HRSC strips without bundle adjustment, we identified 515 cases with obvious high frequent oscillations. In some strips the oscillations might be hidden by other effects, so that the actual number of affected strips might be higher.

All strips with oscillations have been adjusted with a cOPD and with a vOPD for a comparison of both methods. Fig. 6 depicts the precision of the EO after a bundle adjustment with a cOPD (blue) and with a vOPD (green). In the lower range (MIE $<20 \mathrm{~m}$ ), green points occur more often. In contrast the blue points occur more often above $40 \mathrm{~m}$. This clearly depicts an improvement of the EO.

The intersection error maps have been visually inspected for oscillations again. With a cOPD the bundle adjustment was not able to properly model the oscillations in 276 out of the 515 cases. With a vOPD this number was reduced to 8 . In these eight cases the number of tie points was very low because of areas with extremely poor texture showing an icy or dusty surface. Table 2 summaries the results.

\begin{tabular}{llll}
\hline & no BA & BA (cOPD) & BA (vOPD) \\
\hline strips w. oscillations & 515 & 276 & 8 \\
MIE [m] & 43.3 & 27.1 & 17.2 \\
\hline
\end{tabular}

Table 2: Results of 515 HRSC image strips for different bundle adjustment (BA) runs

The average mean intersection error of all 515 strips was $43.3 \mathrm{~m}$ before the adjustment. Compared to the mean nadir image resolution of $17.4 \mathrm{~m}$, it does not fulfill the expectations of a precise photogrammetric point determination, even considering the lower resolution of the other channels. The bundle adjustment reduces the intersection error to $27.1 \mathrm{~m}$ using a cOPD and to $17.2 \mathrm{~m}$ using a vOPD, respectively. As expected a vOPD performs out the COPD, and allows an accurate orbit reconstruction for almost all strips, despite of oscillations and partly weak image information.

\subsection{Global data}

To derive a general statement how the bundle adjustment improves the EO, the global HRSC data set was processed. The evaluation of the results are presented in the following. The used images have been taken in the time span between January 9th, 2004 and December 6th, 2015. In this period (orbit numbers 8 to 15,127 ) altogether 4,433 HRSC images have been recorded. This number includes imagery of Phobos and Deimos, limb observations and other strips not usable for our photogrammetric mapping purposes of the Martian surface. Deducting unusable strips the bundle adjustment was carried out on 3,828 data sets and yielded an improvement of the EO in 3,686 cases $(96,3 \%)$ The improvement showed up in two ways: 1) It reduces the mean intersection error, on average by a factor of 2.0. 2) It reduces the systematic height offset to MOLA from $40.1 \mathrm{~m}$ to $4.2 \mathrm{~m}$ (mean of the absolute values). Fig. 7 depicts a histogram of the strip results with respect to the mean intersection error, computed with the nominal EO (red) and with the adjusted EO (green). An obvious shift of the histogram to the left shows the reduced mean intersection error, as a result of the improved EO parameters.

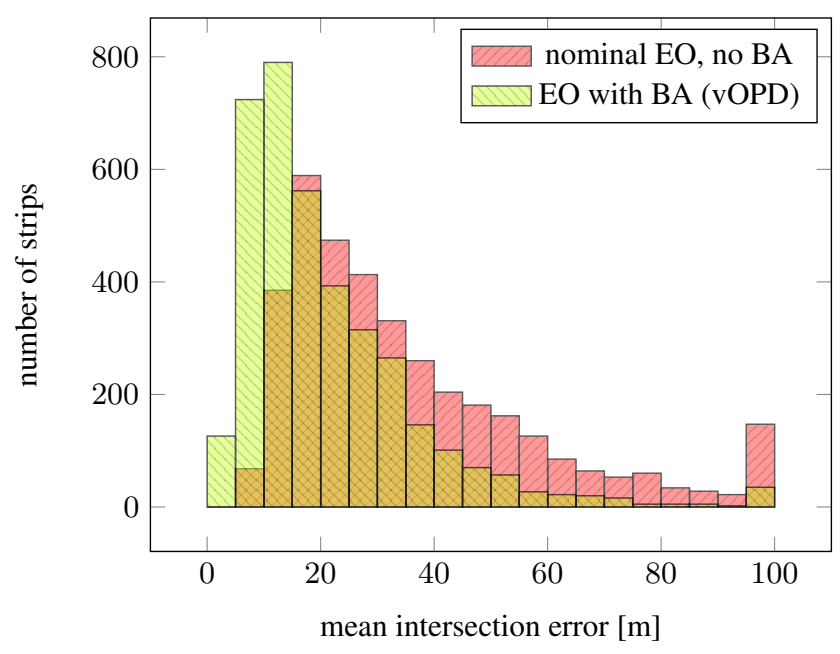

Figure 7: Histogram of 3,686 HRSC image strips with respect to mean intersection error

The second evaluation step validates the fit of the EO to the MOLA DTM. The height difference to the reference surface was computed for a large number of HRSC object points and mapped as depicted in Fig. 8. This mapping allows to recognize local deviations. A reduction of systematic offsets is unveiled by the mean values.

Fig. 9 depicts the histogram of mean height offset. The concentration around $0 \mathrm{~m}$ of the green solution shows an obvious improvement, when using the adjusted EO.

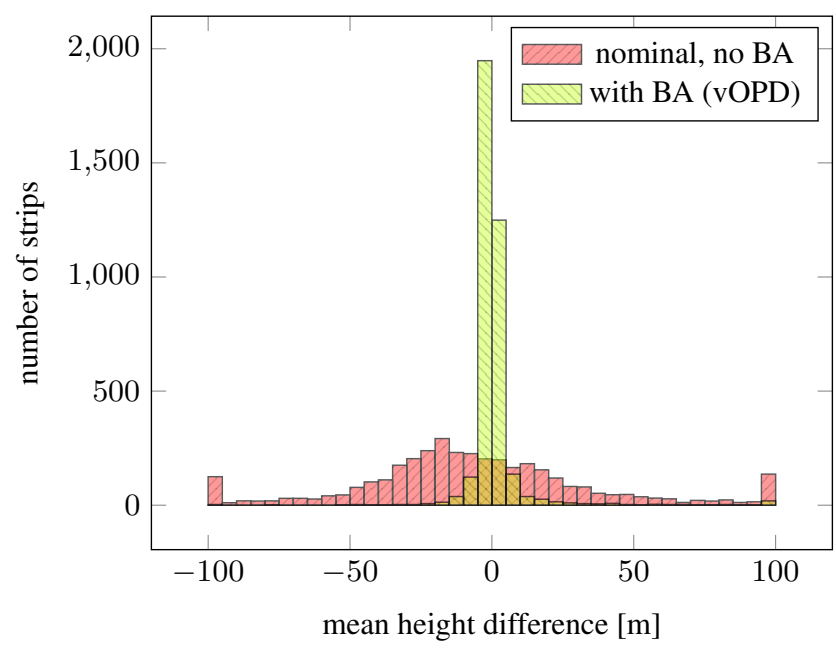

Figure 9: Histogram of 3,686 HRSC image strips with respect to mean MOLA height offset

In Tab. 3 these numbers are summarized by averaging over all HRSC image strips.

\begin{tabular}{lll}
\hline & without BA & with BA \\
\hline MIE[m] & 37.0 & 21.5 \\
mean MOLA Difference[m] & 40.1 & 4.2 \\
\hline
\end{tabular}

Table 3: Improvement of the EO of 3,686 HRSC data sets via bundle adjustment with a vOPD 


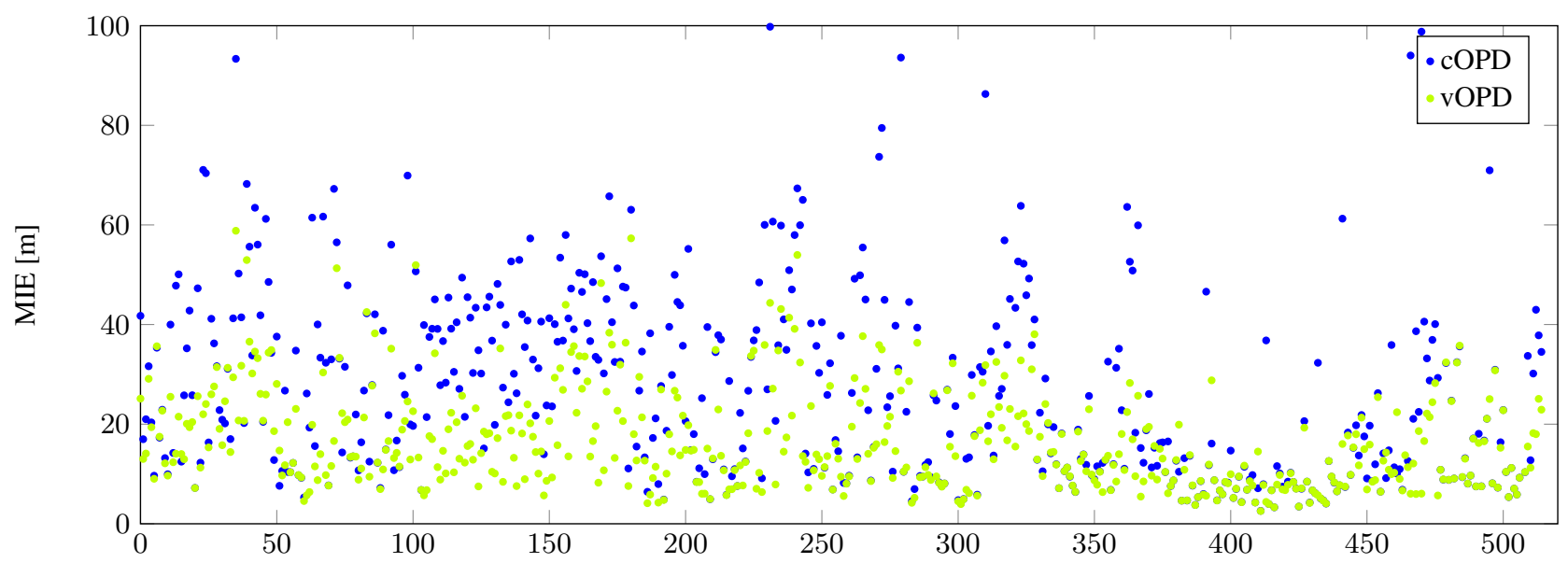

Figure 6: Mean intersection error (MIE) of 515 HRSC image strips with oscillations via bundle adjustment with a constant and a variable orientation point distance

\section{CONCLUSION AND FUTURE WORK}

We improved the concept of OP for modeling the EO of line scanner imagery within a bundle adjustment by varying the OPD according to the available image information given by the number of tie points. In this way the bundle adjustment of HRSC image strips was able to remove image disturbances caused by oscillations of the attitude, not described in the angular elements of the nominal EO. The concept of vOPD can be transferred to any application of a line scanner. It allows the modeling of any high frequent effects in the EO. This is especially required when no observations of an IMU or from GPS/GNSS are available.

Furthermore we performed an experimental study on how the density of tie points affects the quality of orbit modeling. We quantified the ability of this method on a set 515 HRSC image strips with oscillations. We were able to successfully process 96\% (3686 of 3828) of the available HRSC data sets in a fully automated way. The bundle adjustment reduced the forward intersection error of HRSC object points from $37.0 \mathrm{~m}$ to $21.5 \mathrm{~m}$ on average. The systematic height differences to the MOLA reference DTM were reduced on average from $40.1 \mathrm{~m}$ to $4.2 \mathrm{~m}$. The adjusted EO will be used to produce high quality DTMs and ortho images and will be applied as input information for the processing of large HRSC mosaics.

In future work we are interested to investigate the effects of introducing individual OPD for each of the EO elements. Considering the typically stable orbit of a space craft this would reduce the number of unknown parameter in the adjustment significantly without loosing the ability of a detailed modeling of individual elements of attitude. In terms of the processing of HRSC imagery our next research aims at improving the systematic bundle adjustment of very large blocks with the images of many strips combined in a simultaneous adjustment.

\section{ACKNOWLEDGEMENTS}

We thank the HRSC Experiment Teams at DLR Berlin and Freie Universität Berlin as well as the Mars Express Project Teams at ESTEC and ESOC for their successful planning and acquisition of data as well as for making the processed data available to the HRSC Team. This work is funded by the German Federal Ministry for Economic Affairs and Energy (BMWi) within the project management of the German Aerospace Center (DLR) under grant no. 50 QM 1304 . This support is gratefully acknowledged.

\section{REFERENCES}

Albertz, J., Attwenger, M., Barrett, J., Casley, S., Dorninger, P., Dorrer, E., Ebner, H., Gehrke, S., Giese, B., Gwinner, K., Heipke, C., Howington-Kraus, E., Kirk, R., Lehmann, H., Mayer, H., Muller, J., Oberst, J., Ostrovskly, A., Renter, J., Reznik, S., Schmidt, R., Scholten, F., Spiegel, M., Stilla, U., Wählisch, M., Neukum, G. and Team, T. H. C.-I., 2005. HRSC on Mars Express - Photogrammetric and cartographic research. Photogrammetric Engineering \& Remote Sensing 71(10), pp. 1153-1166.

Bostelmann, J. and Heipke, C., 2011. Modeling spacecraft oscillations in HRSC images of Mars Express. In: International Archives of the Photogrammetry, Remote Sensing and Spatial Information Sciences, Vol. 38-4/W19, pp. 51-56.

Bostelmann, J. and Heipke, C., 2014. Analyzing a block of HRSC image strips for a simultaneous bundle adjustment. In: ISPRS Annals of Photogrammetry, Remote Sensing and Spatial Information Sciences, Vol. 2-4, pp. 15-20.

Bostelmann, J., Schmidt, R. and Heipke, C., 2012. Systematic bundle adjustment of HRSC image data. In: International Archives of the Photogrammetry, Remote Sensing and Spatial Information Sciences, Vol. 39-B4, pp. 301-306.

Dowman, I. and Dolloff, J. T., 2000. An evaluation of rational functions for photogrammetric restitution. Photogrammetric Engineering \& Remote Sensing XXXIII, pp. 254-266.

Fraser, C. S. and Hanley, H. B., 2003. Bias Compensation in Rational Functions for Ikonos Satellite Imagery. Photogrammetric Engineering \& Remote Sensing 69(1), pp. 53-57.

Gwinner, K., Jaumann, R., Bostelmann, J., Dumke, A., Elgner, S., Heipke, C., Kersten, E., Michael, G., Preusker, F., Roatsch, T., Schmidt, R., Scholten, F., Spiegel, M., van Gasselt, S., Walter, S. and the HRSC Global Topography and Mosaic Generation Task Group, 2015. The first Quadrangle of the Mars Express HRSC Multi-Orbit Data Products (MC-11-E). In: European Planetary Science Congress EPSC2015-672, Vol. 10, pp. 11-12.

Gwinner, K., Jaumann, R., Hauber, E., Hoffmann, H., Heipke, C., Oberst, J., Neukum, G., Ansan, V., Bostelmann, J., Dumke, A., Elgner, S., Erkeling, G., Fueten, F., Hiesinger, H., Hoekzema, N., Kersten, E., Loizeau, D., Matz, K.-D., McGuire, P., Mertens, V., Michael, G., Pasewaldt, A., Pinet, P., Preusker, F., Reiss, D., Roatsch, T., Schmidt, R., Scholten, F., Spiegel, M., Stesky, R., Tirsch, D., van Gasselt, S., Walter, S., Wählisch, M. and Willner, K., 2016. The High Resolution Stereo Camera (HRSC) of Mars 


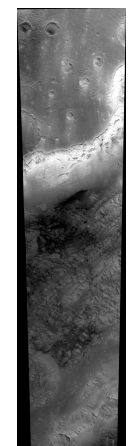

h0259

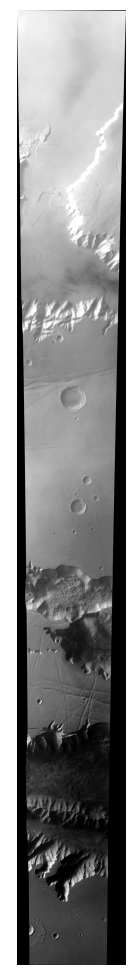

h0887

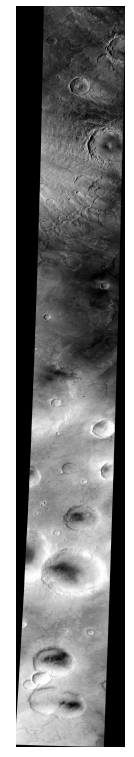

$\mathrm{h} 4330$
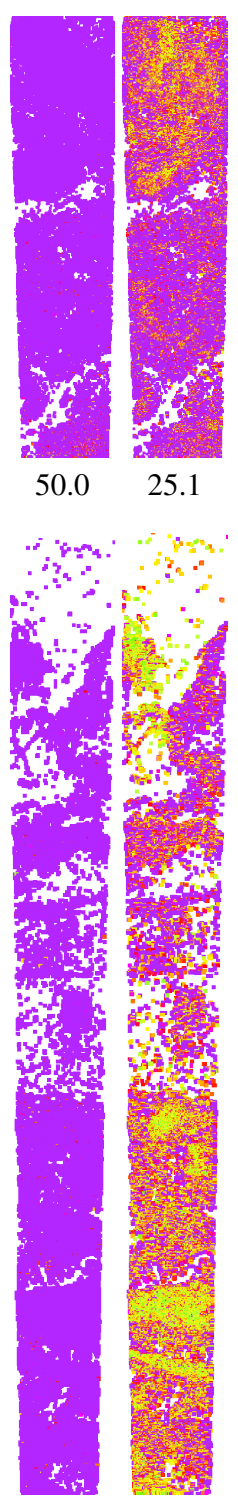

$$
38.2
$$

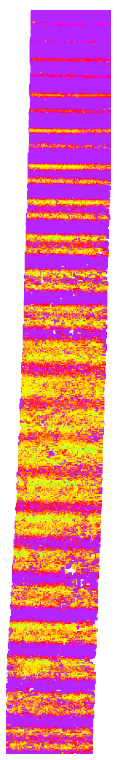

19.7
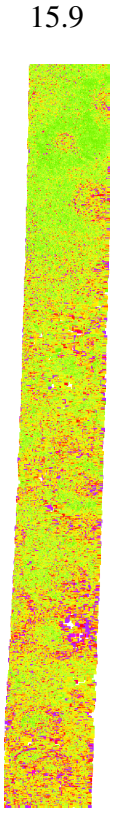
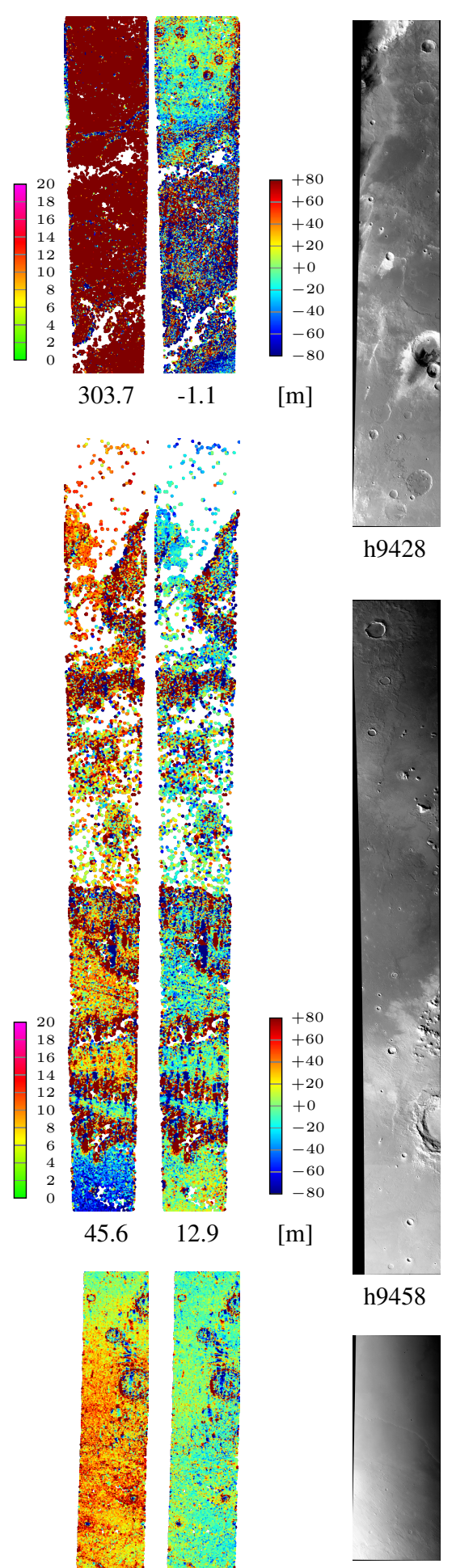

$\mathrm{hb} 472$

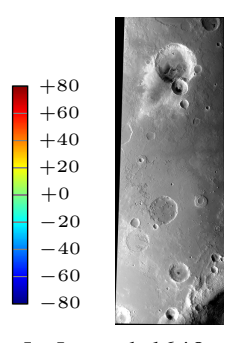

[m] hd640
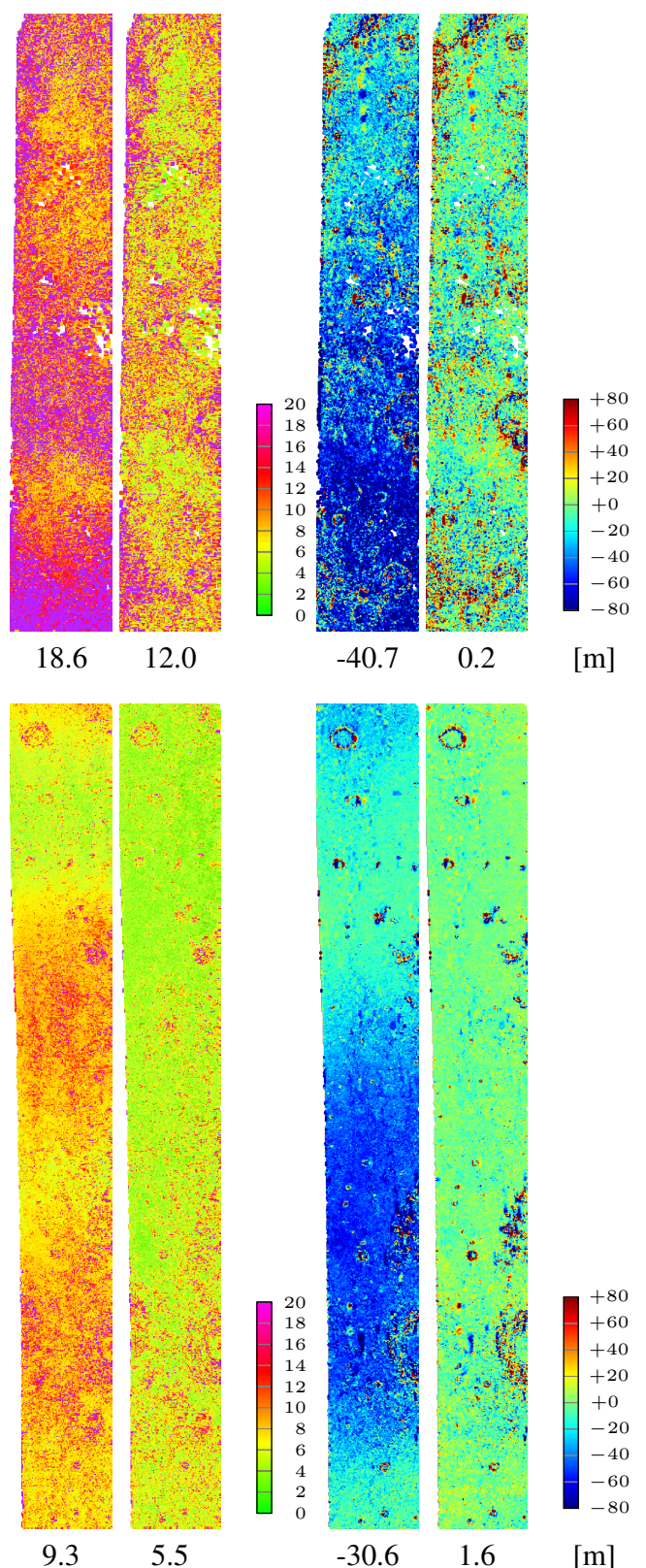

$[\mathrm{m}]$
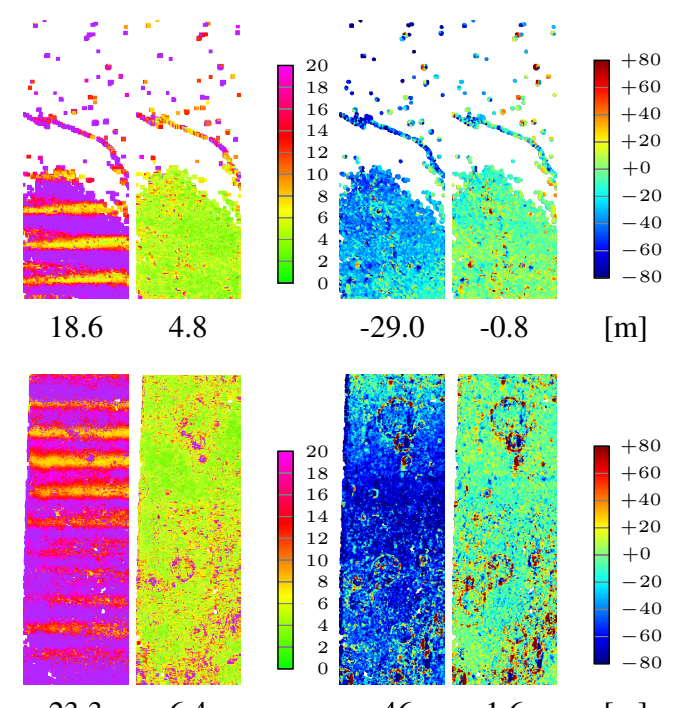

$[\mathrm{m}]$

Figure 8: Evaluation of bundle adjustment (BA) results for 7 out of 3686 HRSC data sets as examples. Each from left to right: 1) nadir ortho image with orbit number; error maps with mean values for: intersection error 2) without BA and 3) with BA (vOPD), height differences between HRSC points and MOLA DTM 4) without BA and 5) with BA (vOPD). 
Express and its Approach to Science Analysis and Mapping for Mars and its Satellites. Planetary and Space Science (In Press).

Gwinner, K., Scholten, F., Preusker, F., Elgner, S., Roatsch, T., Spiegel, M., Schmidt, R., Oberst, J., Jaumann, R. and Heipke, C., 2010. Topography of Mars from global mapping by HRSC highresolution digital terrain models and orthoimages: Characteristics and performance. Earth and Planetary Science Letters 294(3-4), pp. 506-519.

Gwinner, K., Scholten, F., Spiegel, M., Schmidt, R., Giese, B., Oberst, J., Heipke, C., Jaumann, R. and Neukum, G., 2009. Derivation and Validation of High-Resolution Digital Terrain Models from Mars Express HRSC Data. Photogrammetric Engineering \& Remote Sensing 75(9), pp. 1127-1142.

Heipke, C., Oberst, J., Albertz, J., Attwenger, M., Dorninger, P., Dorrer, E., Ewe, M., Gehrke, S., Gwinner, K., Hirschmüller, H., Kim, J. R., Kirk, R. L., Mayer, H., Muller, J. P., Rengarajan, R., Rentsch, M., Schmidt, R., Scholten, F., Shan, J., Spiegel, M., Wählisch, M. and Neukum, G., 2007. Evaluating planetary digital terrain models-The HRSC DTM test. Planetary and Space Science 55, pp. 2173-2191.

Hofmann, O., Nave, P. and Ebner, H., 1982. DPS - A Digital Photogrammetric System for Producing Digital Elevation Models and Orthophotos by Means of Linear Array Scanner Imagery. In: International Archives of the Photogrammetry, Remote Sensing and Spatial Information Sciences, Vol. 24 (3), pp. 216-227.

Hu, Y. and Tao, C. V., 2002. Updating solutions of the rational function model using additional control information. Photogrammetric Engineering \& Remote Sensing 68(7), pp. 715-723.

Jaumann, R., Neukum, G., Behnke, T., Duxbury, T. C., Eichentopf, K., Flohrer, J., Gasselt, S. v., Giese, B., Gwinner, K., Hauber, E., Hoffmann, H., Hoffmeister, A., Köhler, U., Matz, K. D., McCord, T. B., Mertens, V., Oberst, J., Pischel, R., Reiss, D., Ress, E., Roatsch, T., Saiger, P., Scholten, F., Schwarz, G., Stephan, K. and Wählisch, M., 2007. The high-resolution stereo camera (HRSC) experiment on Mars Express: Instrument aspects and experiment conduct from interplanetary cruise through the nominal mission. Planetary and Space Science 55(7), pp. 928 952.

Jaumann, R., Tirsch, D., Hauber, E., Ansan, V., Di Achille, G., Erkeling, G., Fueten, F., Head, J., Kleinhans, M., Mangold, N., Michael, G., Neukum, G., Pacifici, A., Platz, T., Pondrelli, M., Raack, J., Reiss, D., Williams, D., Adeli, S., Baratoux, D., de Villiers, G., Foing, B., Gupta, S., Gwinner, K., Hiesinger, H., Hoffmann, H., Deit, L. L., Marinangeli, L., Matz, K.-D., Mertens, V., Muller, J., Pasckert, J., Roatsch, T., Rossi, A., Scholten, F., Sowe, M., Voigt, J. and Warner, N., 2015. Quantifying geological processes on Mars -Results of the high resolution stereo camera (HRSC) on Mars express. Planetary and Space Science 112, pp. 53-97.

Kornus, W., 1999. Dreidimensionale Objektrekonstruktion mit digitalen Dreizeilenscannerdaten des Weltraumprojekts MOMS02/D2. PhD thesis, DGK-C, Nr. 496, Deutsche Geodätische Kommission, Verlag C. H. Beck, München.

Michael, G., Walter, S., Kneissl, T., Zuschneid, W., Gross, C., McGuire, P., Dumke, A., Schreiner, B., van Gasselt, S., Gwinner, K. and Jaumann, R., 2016. Systematic processing of Mars Express HRSC panchromatic and colour image mosaics: Image equalisation using an external brightness reference. Planetary and Space Science 121, pp. 18-26.

Müller, F., 1991. Photogrammetrische Punktbestimmung mit Bildern digitaler Dreizeilenkameras. PhD thesis, DGK-C, Nr.
372, Deutsche Geodätische Kommission, Verlag C. H. Beck, München.

Neukum, G., Jaumann, R. and The HRSC Co-Investigator Team, 2004. HRSC: The High Resolution Stereo Camera of Mars express. Mars Express: The Scientific Payload, Eur. Space Agency Spec. Publ., ESA-SP 1240, pp. 17-36.

Ohlhof, T., 1996. Lokale, regionale und globale Punktbestimmung mit Dreizeilenbilddaten und Bahninformationen der Mars 96-Mission. PhD thesis, DGK-C, Nr. 445, Deutsche Geodätische Kommission, Verlag C. H. Beck, München.

Poli, D., 2005. Modelling of spaceborne linear array sensors. $\mathrm{PhD}$ thesis, IGP Report No. 85, Institute of Geodesy and Photogrammetry, ETH Zurich, Switzerland. 204 pages.

Schmidt, R., 2008. Automatische Bestimmung von Verknüpfungspunkten für HRSC-Bilder der Mars ExpressMission. PhD thesis, DGK-C, Nr. 623, Deutsche Geodätische Kommission, Verlag C. H. Beck, München.

Scholten, F., Gwinner, K., Roatsch, T., Matz, K., Wahlisch, M., Giese, B., Oberst, J., Jaumann, R. and Neukum, G., 2005. Mars Express HRSC Data Processing - Methods and Operational Aspects. Photogrammetric Engineering \& Remote Sensing 71(10), pp. 1143-1152.

Smith, D. E., Zuber, M. T., Frey, H. V., Garvin, J. B., Head, J. W., Muhleman, D. O., Pettengill, G. H., Phillips, R. J., Solomon, S. C., Zwally, H. J., Banerdt, W. B., Duxbury, T. C., Golombek, M. P., Lemoine, F. G., Neumann, G. a., Rowlands, D. D., Aharonson, O., Ford, P. G., Ivanov, A. B., Johnson, C. L., McGovern, P. J., Abshire, J. B., Afzal, R. S., Sun, X. and Others, 2001. Mars Orbiter Laser Altimeter: Experiment summary after the first year of global mapping of Mars. Journal of Geophysical Research: Planets 106(E10), pp. 23689-23722.

Spiegel, M., 2007a. Improvement of interior and exterior orientation of the three line camera HRSC with a simultaneous adjustment. In: International Archives of the Photogrammetry, Remote Sensing and Spatial Information Sciences, Vol. 36, pp. 161-166.

Spiegel, M., 2007b. Kombinierte Ausgleichung der Mars Express HRSC Zeilenbilddaten und des Mars Global Surveyor MOLA DGM. PhD thesis, DGK-C, Nr. 610, Deutsche Geodätische Kommission, Verlag C. H. Beck, München.

Strunz, G., 1993. Bildorientierung und Objektrekonstruktion mit Punkten, Linien und Flächen. PhD thesis, DGK-C, Nr. 408, Deutsche Geodätische Kommission.

Weser, T., Rottensteiner, F., Willneff, J., Poon, J. and Fraser, C. S., 2008. Development and testing of a generic sensor model for pushbroom satellite imagery. The Photogrammetric Record 23(123), pp. 255-274. 